\title{
A common approach for absolute quantification of short chain CoA thioesters in prokaryotic and eukaryotic microbes
}

\author{
Lars Gläser ${ }^{1}$, Martin Kuhl', Sofija Jovanovic ${ }^{1}$, Michel Fritz ${ }^{1}$, Bastian Vögeli ${ }^{2}$, Tobias J. Erb², Judith Becker ${ }^{1}$ \\ and Christoph Wittmann ${ }^{1 *}$ (D)
}

\begin{abstract}
Background: Thioesters of coenzyme A participate in $5 \%$ of all enzymatic reactions. In microbial cell factories, they function as building blocks for products of recognized commercial value, including natural products such as polyketides, polyunsaturated fatty acids, biofuels, and biopolymers. A core spectrum of approximately 5-10 short chain thioesters is present in many microbes, as inferred from their genomic repertoire. The relevance of these metabolites explains the high interest to trace and quantify them in microbial cells.
\end{abstract}

Results: Here, we describe a common workflow for extraction and absolute quantification of short chain CoA thioesters in different gram-positive and gram-negative bacteria and eukaryotic yeast, i.e. Corynebacterium glutamicum, Streptomyces albus, Pseudomonas putida, and Yarrowia lipolytica. The approach assessed intracellular CoA thioesters down to the picomolar level and exhibited high precision and reproducibility for all microbes, as shown by principal component analysis. Furthermore, it provided interesting insights into microbial CoA metabolism. A succinyl-CoA synthase defective mutant of C. glutamicum exhibited an unaffected level of succinyl-CoA that indicated a complete compensation by the L-lysine pathway to bypass the disrupted TCA cycle. Methylmalonyl-CoA, an important building block of high-value polyketides, was identified as dominant CoA thioester in the actinomycete S. albus. The microbe revealed a more than 10,000-fold difference in the abundance of intracellular CoA thioesters. A recombinant strain of S. albus, which produced different derivatives of the antituberculosis polyketide pamamycin, revealed a significant depletion of COA thioesters of the ethylmalonyl COA pathway, influencing product level and spectrum.

Conclusions: The high relevance of short chain CoA thioesters to synthetize industrial products and the interesting insights gained from the examples shown in this work, suggest analyzing these metabolites in microbial cell factories more routinely than done so far. Due to its broad application range, the developed approach appears useful to be applied this purpose. Hereby, the possibility to use one single protocol promises to facilitate automatized efforts, which rely on standardized workflows.

Keywords: Corynebacterium glutamicum, Streptomyces albus, Pseudomonas putida, Yarrowia lipolytica, Lysine, Pamamycin, CoA thioester, LC-MS

*Correspondence: christoph.wittmann@uni-saarland.de

${ }^{1}$ Institute of Systems Biotechnology, Saarland University, Saarbrücken, Germany

Full list of author information is available at the end of the article

\section{Background}

Microbial cell factories are a key to the bio-based industry [1]. Upgrading and streamlining their biocatalytic activity through systems metabolic engineering requires detailed understanding of the underlying metabolism [2-5]. Among other techniques, the assessment of intracellular metabolite

c) The Author(s) 2020. This article is licensed under a Creative Commons Attribution 4.0 International License, which permits use, sharing, adaptation, distribution and reproduction in any medium or format, as long as you give appropriate credit to the original author(s) and the source, provide a link to the Creative Commons licence, and indicate if changes were made. The images or other third party material in this article are included in the article's Creative Commons licence, unless indicated otherwise in a credit line to the material. If material is not included in the article's Creative Commons licence and your intended use is not permitted by statutory regulation or exceeds the permitted use, you will need to obtain permission directly from the copyright holder. To view a copy of this licence, visit http://creativeco mmons.org/licenses/by/4.0/. The Creative Commons Public Domain Dedication waiver (http://creativecommons.org/publicdomain/ zero/1.0/) applies to the data made available in this article, unless otherwise stated in a credit line to the data. 
levels and pathway fluxes has proven valuable to understand metabolic network function and its regulation and derive novel targets for strain engineering $[1,6]$.

A relevant group of metabolites are thioesters, esters between a carboxylic acid and a thiol. In microbial metabolism, the best-known and most relevant thioesters are short chain CoA thioesters, derivatives of coenzyme A (CoA) $[7,8]$. Notably, CoA thioesters such as acetyl-CoA and succinyl-CoA participate in 5\% of all enzymatic reactions and at least one-third of all cellular carbon is typically metabolized through a CoA thioester [7]. As example, they provide activated groups to drive the anabolic synthesis of cellular constituents such as peptides, fatty acids, sterols, and terpenes, display intermediates of catabolic pathways, and are essential to central energy metabolism [8]. Today, bioinformatics databases reveal more than two hundred naturally occurring CoA thioester derivatives [8], of which a core spectrum between approximately 5-10 compounds is potentially present in most microbes, based on their genomic repertoire $[9,10]$.

From a commercial perspective, CoA thioesters display building blocks of a wide range of industrially interesting products. Prominent examples are polyketides [11], polyunsaturated fatty acids (PUFAs) [12], polyhydroxyalkanoates (PHAs) [13], biofuels [14], amino acids [15], and dicarboxylic acids [16], among others [17]. This relevance might explain the increasing interest to trace CoA thioesters. Previous efforts have provided different experimental approaches, each specifically designed for a particular microbe, including indirect analysis of $\mathrm{CoA}$ thioesters via measurement of the respective organic acid, isotope dilution and enzymatic assays [17-20].

In this work, we have set up a sensitive, robust, and reproducible workflow to quantify short chain CoA thioesters in microbes. For this purpose, we adapted a previous protocol, used to assess a wide spectrum of CoA thioesters in the methylotrophic bacterium Methylobacterium extorquens [21]. After improvement and careful validation, we demonstrated the approach for industrially relevant microorganisms, which utilize CoA thioesters to form value-added products: the gram-positive bacteria Corynebacterium glutamicum [15], and Streptomyces albus [11], the gram-negative bacterium Pseudomonas putida [13], and the eukaryotic yeast Yarrowia lipolytica [12].

\section{Results}

\section{Set up and validation of a single protocol for extraction and quantification of short chain CoA thioesters in gram-positive and gram-negative bacteria and eukaryotic yeast}

A synthetic mixture of $11 \mathrm{CoA}$ thioesters of interest was used to set up a chromatographic method. Using a porous organo-silica reversed phase column $(100 \times 2.1 \mathrm{~mm}$,
$1.5 \mu \mathrm{m}$ ), efficient separation of the analytes was achieved within $25 \mathrm{~min}$, including the isobaric derivatives succinyl-CoA/methylmalonyl-CoA and methylsuccinyl-CoA/ ethylmalonyl-CoA, respectively (Additional file 1: Fig. S1). As exception butyryl-CoA and isobutyryl-CoA coeluted in all cases tested (data not shown). They could not be distinguished in the MS due to their identical mass either and were therefore regarded as one pool. The linear range for quantification covered 5-8 orders of magnitude, down to the picomolar level (Additional file 1: Fig. S2).

Next, we aimed to develop one common workflow, which was suitable to analyze real samples from the different microbes. Initial tests with C. glutamicum revealed that combined quenching and extraction was straightforward to handle experimentally and provided extracts of reproducible quality (data not shown), so that we used it as a starting point for development. Several practical challenges resulted from the nature of the different microbes and the used culture conditions and had to be addressed.

First, the chosen small column geometry and particle size turned out incompatible with certain samples. Over rather few injections (10-20), the column pressure increased from initially 250 to 1000 bar, which required extensive cleaning with water to regenerate the separation column. However, despite such efforts, we faced a rapid loss of separation efficiency. This was especially true for samples of $S$. albus and C. glutamicum, grown in media with elevated ionic strength. The use of a larger column $(100 \times 4.6 \mathrm{~mm})$ and a twofold increased particle size of the separation material $(3 \mu \mathrm{m})$ solved this issue so that more than 500 samples could be analyzed on the same column without pressure increase and loss in separation performance, independent of the microbe investigated. Due to the larger geometry, the eluent flow was increased to $600 \mu \mathrm{L} \mathrm{min}{ }^{-1}$, which kept the analysis time at $25 \mathrm{~min}$ and provided a robust approach for the analytics. By far the best separation was achieved using a core-shell silica column instead of the porous silica column (Fig. 1). Due to superior properties of the coreshell material, the resulting peaks were narrower so that the gradient could be increased substantially. Altogether, this shortened the analysis time for all CoA esters to only $10 \mathrm{~min}$ (Fig. 1a). The analysis of cell extracts yielded clean chromatograms with high signal quality, even for low abundance thioesters (Fig. 1 b, c).

Second, small biomass amounts, typically chosen for sampling in metabolomics due to easier handling, were not suitable to precisely quantify all $\mathrm{CoA}$ thioesters present in vivo due to an extremely low abundance of some of them. As example, S. albus contained ultralow amounts of crotonyl-CoA, which yielded low quality 


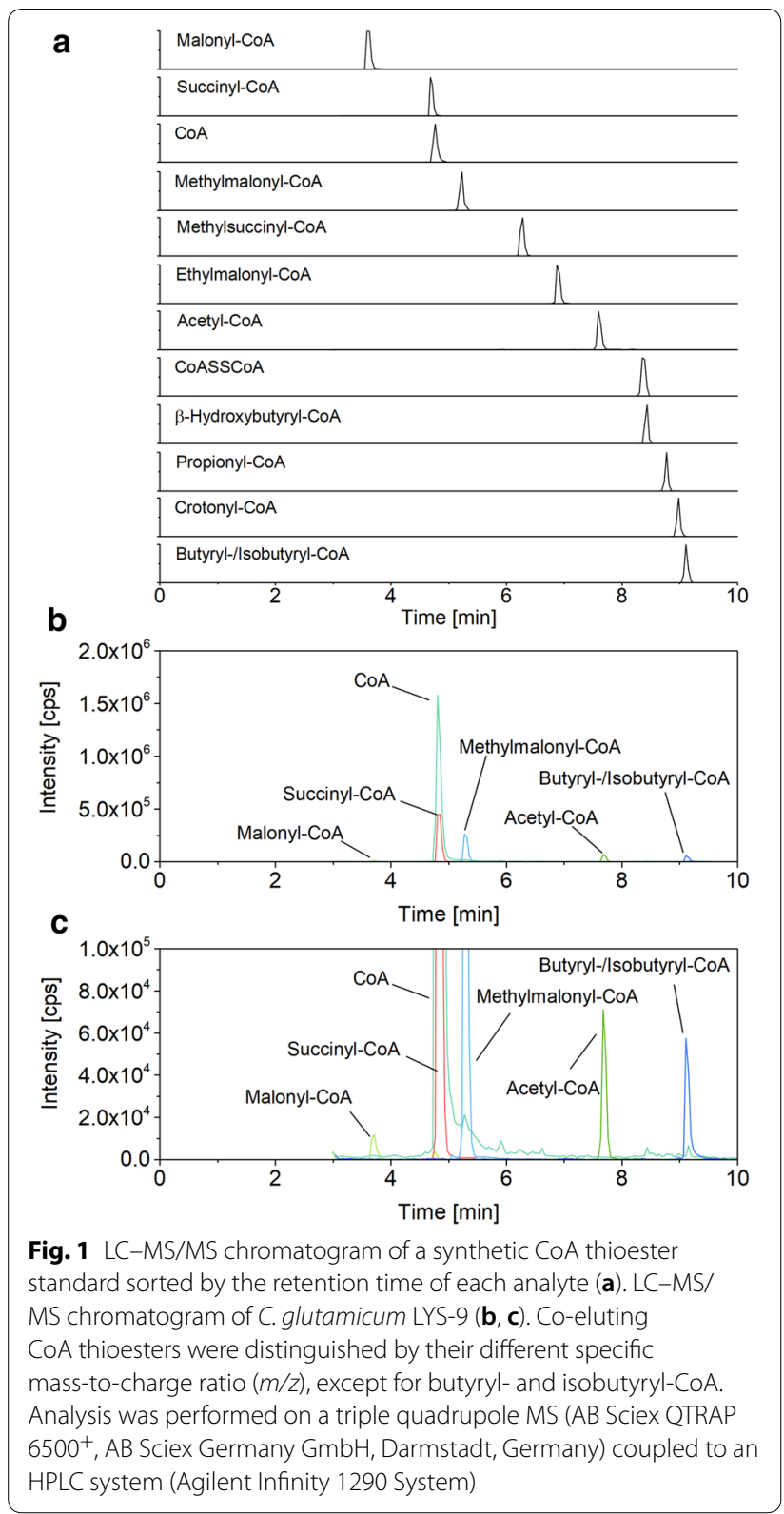

signals near the threshold, when extracted from $0.6 \mathrm{mg}$ biomass. Similar observations were made for the other microbes studied. An increase of the sample amount to $8 \mathrm{mg}$, however, allowed clean detection and quantification of all CoA thioesters to be expected from the genomic repertoire for each of the tested strains and conditions.

Third, the higher biomass amounts caused difficulties in dissolving lyophilized extracts, after freezedrying them together with extracted cell fragments. The obtained solutions were too viscous, especially when sampling the filamentous actinomycete, to appropriately filter them prior to analysis. Due to this, we introduced a centrifugation step between extraction and lyophilization, which allowed a better handling, especially for $S$. albus. Additional tests for all strains revealed that after two washing cycles the cell pellets did not contain any significant residuals of the analytes of interest, which ensured complete extraction. In the following, ${ }^{13} \mathrm{C}$ labeled cell extracts were prepared by growing each microbe on its corresponding $\left[\mathrm{U}_{-}{ }^{13} \mathrm{C}\right]$ substrate and conducting the established sample processing. The concentration of the ${ }^{13} \mathrm{C}$ CoA thioesters was precisely quantified against synthetic standards so that the ${ }^{13} \mathrm{C}$ extracts could then be used to quantify absolute concentrations.

Fourth, free CoA underwent dimerization to a certain degree during the sample processing. When analyzing the synthetic standard, approximately $15 \% \pm 3 \%$ of free coenzyme A was observed as CoA-disulfide (CoA-S-SCoA), eluting 3.5 min after the free monomer (Fig. 1, Additional file 1: Table S1). This phenomenon was also observed for cell extract samples.

\section{C. glutamicum reveals a small spectrum of CoA thioesters with methylmalonyl-CoA as the dominating metabolite}

The L-lysine producing mutant C. glutamicum LYS-9 was analyzed during batch growth on glucose. It continuously accumulated L-lysine to a final titer of $10.5 \mathrm{mM}$ at a yield of $190 \mathrm{mmol} \mathrm{mol}^{-1}$ (Fig. 2a). The specific growth rate remained constant over the whole cultivation $\left(\mu=0.27 \mathrm{~h}^{-1}\right)$. The cell interior of C. glutamicum LYS-9 contained five CoA thioesters: acetyl-CoA, malonyl-CoA, methylmalonyl-CoA, succinyl-CoA, and butyryl/isobutyryl-CoA. The esters differed almost 200 -fold in abundance. Methylmalonyl-CoA exhibited the highest concentration (up to $750 \mathrm{nmol} \mathrm{g} \mathrm{g}^{-1}$ ), followed by succinyl-CoA (110 nmol $\left.\mathrm{g}^{-1}\right)$, malonylCoA $\left(30 \mathrm{nmol} \mathrm{g}^{-1}\right)$, acetyl-CoA $\left(5 \mathrm{nmol} \mathrm{g}^{-1}\right)$, and butyryl/isobutyryl-CoA $\left(3 \mathrm{nmol} \mathrm{g}^{-1}\right)$. Additionally, free coenzyme A was observed in significant amount $\left(820 \mathrm{nmol} \mathrm{g}^{-1}\right)$. The level of all CoA thioesters remained stable over time, except for malonyl-CoA, which decreased by approximately $50 \%$ in later cultivation stages (Fig. 2c, d). Incubated under the same conditions as its ancestor, C. glutamicum LYS-9 $\triangle$ sucCD (lacking succinyl-CoA synthetase) formed $12 \mathrm{mM} \mathrm{L}$-lysine at an increased yield of $243 \mathrm{mmol} \mathrm{mol}^{-1}$, while growing at a specific growth rate of $\mu=0.25 h^{-1}$ (Fig. 2b). The spectrum of intracellular CoA thioesters was almost unaffected in the TCA-cycle defective mutant, as compared to LYS-9. This was also true for succinyl-CoA, the substrate of the deleted enzyme. Its pool size was identical 

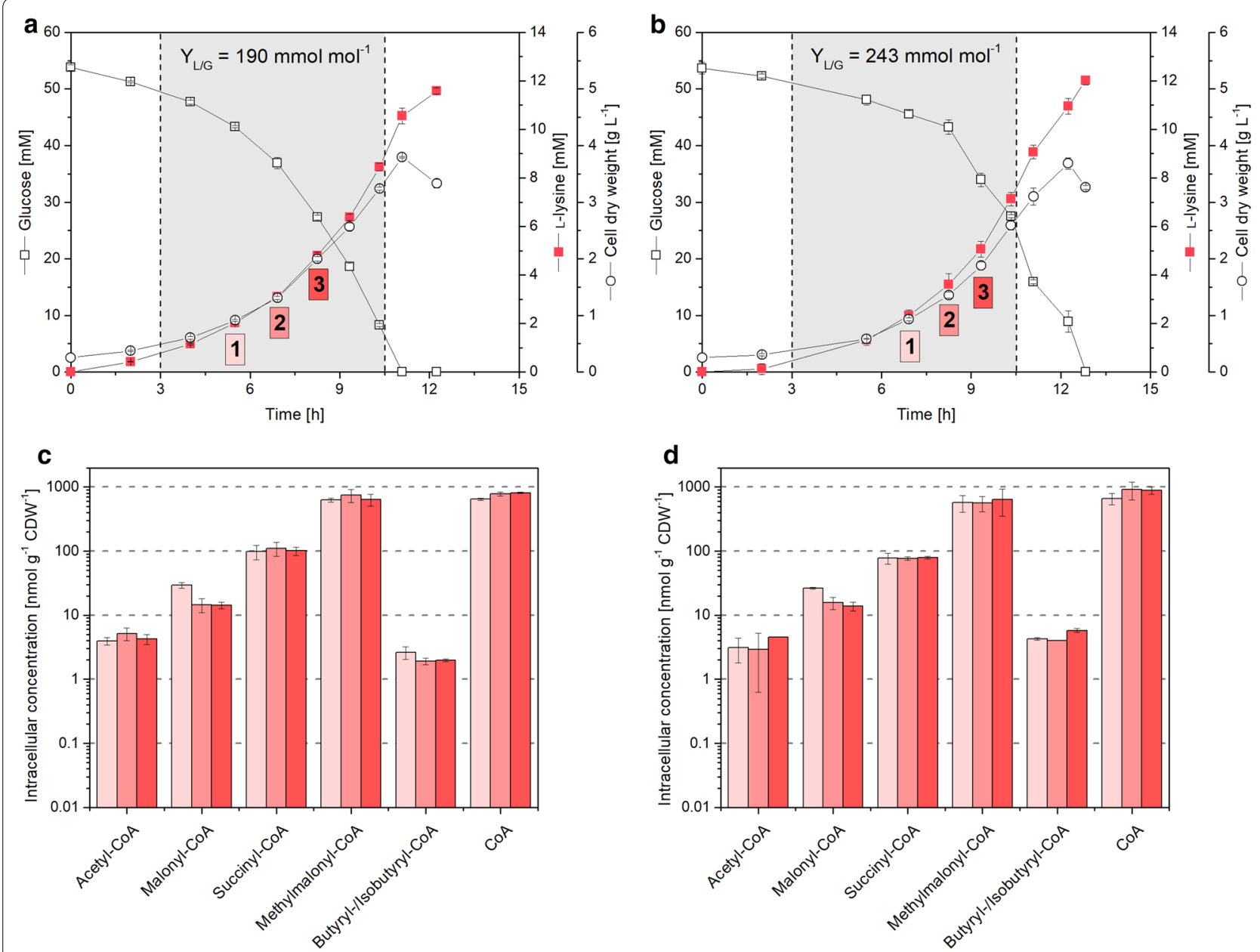

Fig. 2 Absolute quantification of intracellular CoA thioesters in L-lysine producing Corynebacterium glutamicum LYS-9 (left) and LYS-9 $\triangle$ sucCD (right) during growth on glucose. The data comprise the time profile of glucose, $L-l y s i n e$, and cell concentration $(\mathbf{a}, \mathbf{b})$ and absolute levels of intracellular CoA thioesters (c, $\mathbf{d})$. The yield for L-lysine reflects the major production phase, indicated by the gray area, and analyzed for free CoA and CoA thioesters at three time points. The data for these time points are shown in subfigures $\mathbf{c}$ and $\mathbf{d} . \mathrm{n}=3$

in both strains. Only butyryl/isobutyryl-CoA slightly differed (Student's $t$ test, $p=0.01$ ).

\section{The actinomycete S. albus exhibits a rich set of CoA thioesters varying more than 10,000 -fold in intracellular availability}

The wild type S. albus J1074 was grown on mannitolbased minimal medium (Fig. 3a). The substrate was consumed over a time of $25 \mathrm{~h}$ and cells reached a cell dry weight of $4.5 \mathrm{~g} \mathrm{~L}^{-1}$. CoA thioesters were sampled at three time points during the mid-growth phase. The actinomycete revealed a rich spectrum of eleven $\mathrm{CoA}$ thioesters with side chains of two, three, four and five carbons (Fig. 3c). Acetyl-CoA was most abundant (up to $230 \mathrm{nmol} \mathrm{g}^{-1}$ ), followed by succinyl-CoA, malonyl-CoA, and butyryl/isobutyryl-CoA. The other six thioesters partly exhibited much lower levels. Crotonyl-CoA and
$\beta$-hydroxybutyryl-CoA were contained only in trace amounts down to $0.3 \mathrm{nmol} \mathrm{g}^{-1}$. Furthermore, S. albus contained free coenzyme A up to $60 \mathrm{nmol} \mathrm{g}^{-1}$. Along the cultivation, most pools (including those of high abundance) remained stable, but selected CoA thioesters changed to some extent. As example, the level of the carbon-five side-chain esters ethylmalonyl-CoA and methylsuccinyl-CoA increased over time.

The recombinant strain S. albus J1074/R2 produced $1.3 \mathrm{mg} \mathrm{L}^{-1}$ total pamamycin during growth on mannitol (Fig. 3b). The polyketide was produced from early on, accumulated in an exponential manner during the first hours and levelled off toward the end. The mutant revealed the same number of CoA thioesters as its ancestor S. albus J1074, but strongly differed in amount for some of them. As example, the level of crotonyl CoA was decreased up to more 

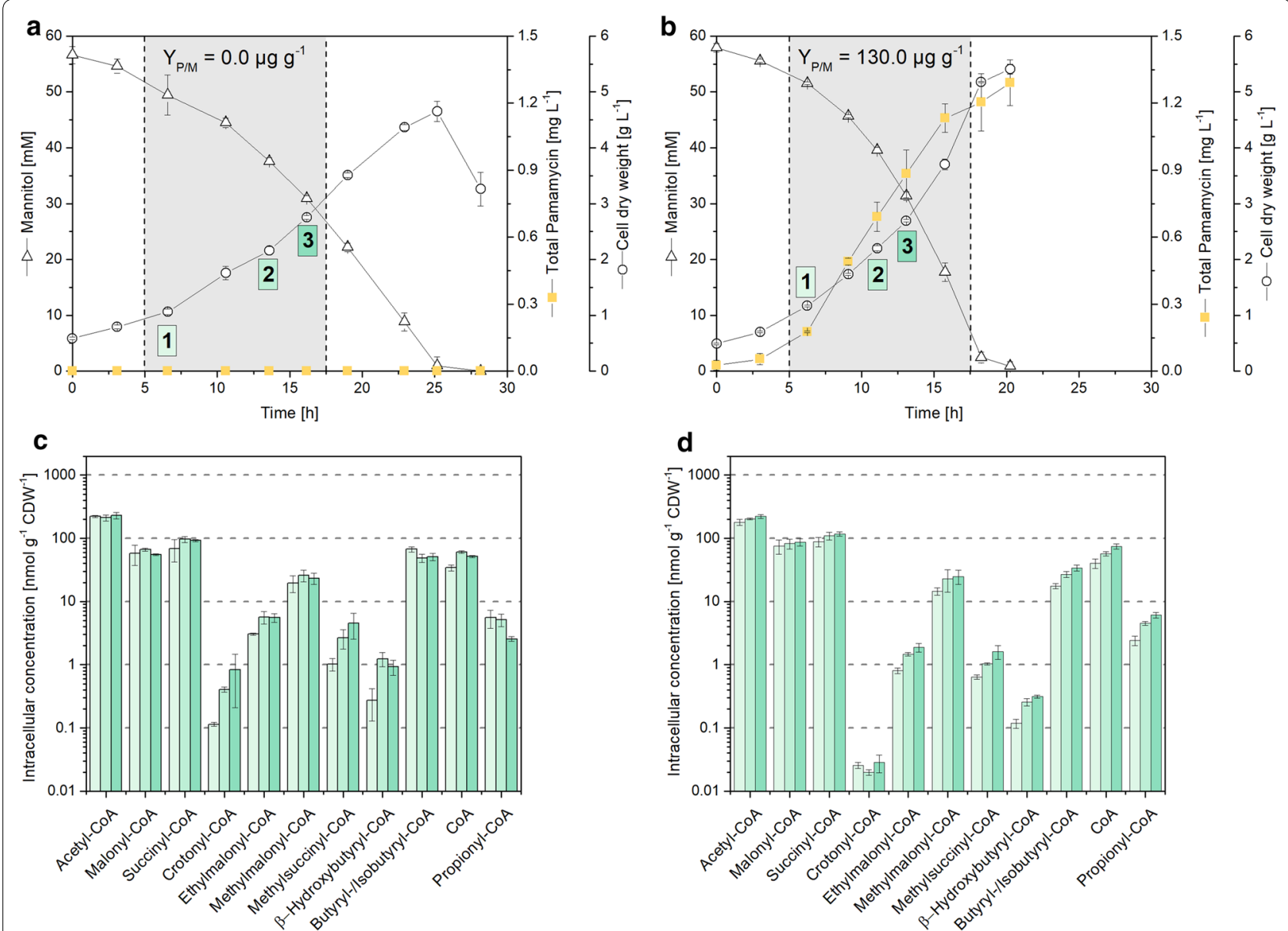

Fig. 3 Absolute quantification of intracellular CoA thioesters in Streptomyces albus J1074 (left) and its pamamycin producing mutant J1074/R2 (right) during growth on mannitol. The data comprise the time profile of mannitol, total pamamycin and cell concentration (a, $\mathbf{b})$, and absolute levels of intracellular CoA thioesters and free $\operatorname{CoA}(\mathbf{c}, \mathbf{d})$. The yield for pamamycin reflects the major production phase, indicated by the gray area, and analyzed for CoA thioesters at three time points. $n=3$

than ten-fold to $0.02 \mathrm{nmol} \mathrm{g}^{-1}$. In addition, the levels of $\beta$-hydroxybutyryl-CoA, ethylmalonyl-CoA, and methylsuccinyl-CoA were reduced up to five-fold (Fig. 3c, d). The other CoA thioesters, including pools of highest abundance (acetyl-CoA, malonyl-CoA, succinyl-CoA) appeared relatively unaffected by pamamycin production. Regarding the pamamycin spectrum, the strain synthetized various derivatives that differed in their mass, due to divergent side chains (Pam 579, Pam 593, Pam 607, Pam 621, Pam 635, Pam 649, Pam 663), which is known to be caused by the alternative incorporation of three-carbon malonyl-CoA, fourcarbon methyl-malonyl-CoA, and five-carbon ethylmalonyl-CoA during biosynthesis. At the end of the process, the distribution was Pam 579 (1.5\%), Pam 593 (5.6\%), Pam 607 (40.5\%), Pam 622 (48.1\%), Pam 635 (3.9\%), Pam 649 (0.3\%) and Pam 663 (0.0\%).

\section{Glucose-grown P. putida KT2440 shows a high abundance of free coenzyme $A$ up to 1000 -fold more than bound CoA thioesters}

When grown on glucose, $P$. putida KT2440 contained six intracellular CoA thioesters with two, three and four carbon side chains, respectively: acetyl-CoA, malonylCoA, succinyl-CoA, $\beta$-hydroxybutyryl-CoA, butyryl/ isobutyryl-CoA, and crotonyl-CoA. The level of the CoA thioesters ranged from $280 \mathrm{nmol} \mathrm{g}^{-1}$ (succinyl-CoA) to $1 \mathrm{nmol} \mathrm{g}^{-1}$ (crotonyl-CoA). P. putida KT2440 contained a huge amount of free coenzyme $\mathrm{A}\left(1,260 \mathrm{nmol} \mathrm{g}^{-1}\right)$, 
(See figure on next page.)

Fig. 4 Impact of environmental and genetic perturbation on the spectrum of short-chain CoA thioesters and free coenzyme A in different microbes. The data show direct correlations in absolute CoA thioester levels between different strains of Corynebacterium glutamicum (a, b), Streptomyces albus (c, d), and Pseudomonas putida (e, $\mathbf{f})$, and between glucose and glycerol grown Yarrowia lipolytica $(\mathbf{g}, \mathbf{h})$. The analysis comprised $C$. glutamicum LYS-9 and its succinyl-CoA synthetase deletion mutant LYS-9 $\triangle$ suCCD, which achieved a higher L-lysine yield, due to flux coupling of the L-lysine pathway with the disrupted TCA cycle $(\mathbf{a}, \mathbf{b})$ [15]. In comparison to the wild type S. albus J1074, the recombinant producer J1074/R2 formed the polyketide pamamycin from CoA thioester building blocks malonyl-CoA, methylmalonyl-CoA, ethylmalonyl-CoA, succinyl-CoA, and acetyl-CoA (c, d) [1 1]. In addition, the data comprise Pseudomonas putida KT2440 and its glucose dehydrogenase deficient mutant KT2440 $\triangle$ gcd (e, f) [13], and Yarrowia lipolytica Po1h::Af4 using glucose and glycerol as sole carbon source $(\mathbf{g}, \mathbf{h})$ [12]. The statistical significance for observed differences in CoA thioester levels (t-test $p<0.05$ ) is marked by an asterisk. $\mathrm{n}=3$

exceeding the sum of all thioester pools more than twofold and the level of individual thioesters up to more than 1000-fold (Fig. 4f). The glucose dehydrogenase $(g c d)$ deletion mutant KT2440 $\Delta g c d$, grown under the same conditions, showed a five-fold decreased level for succinyl-CoA $(\mathrm{p}=0.01)$ and $\beta$-hydroxybutyryl-CoA (0.6fold, $\mathrm{p}=0.01$ ). The most obvious consequence of the $\mathrm{gcd}$ deletion was a dramatically decreased abundance of free coenzyme A (172 nmol g ${ }^{-1}$ ) (Fig. 4f).

\section{Y. lipolytica adapts the level of carbon three thioesters, when grown on glucose and glycerol}

Acetyl-CoA, malonyl-CoA, butyryl/isobutyryl-CoA, $\beta$-hydroxybutyryl-CoA, crotonyl-CoA, and succinyl CoA were present, when the yeast was grown on glucose or on glycerol (Fig. 4h). The carbon source specifically affected the intracellular level of carbon-three CoA thioesters. Whereas malonyl-CoA was significantly increased on glucose (19 $\left.\mathrm{nmol} \mathrm{g}^{-1}\right)$ as compared to glycerol $\left(15 \mathrm{nmol} \mathrm{g}^{-1}\right.$ ) (Student's t-test, $\left.p=0.04\right)$, propionyl-CoA was reduced more than threefold as compared glycerolgrown cells. The other CoA thioesters as well as free CoA showed similar concentrations on both substrates.

\section{Discussion}

The developed experimental workflow enables precise and reproducible quantification of $\mathrm{COA}$ thioesters in gram-positive and gram-negative bacteria and eukaryotic yeast

Thioesters of coenzyme A play an important role in metabolism and participate in $5 \%$ of all enzymatic conversions [7]. However, only selected studies so far have managed to assess their presence in microbial cells using different protocols, specifically elaborated for the given question $[8,20,22,23]$. In this work, we successfully adapted a workflow with integrated quenching and extraction using pre-cooled acetonitrile and formic acid, previously described for the methanol-utilizing bacterium Methylobacterium extorquens [21] to quantitatively extract intracellular CoA thioesters from C. glutamicum, S. albus, P. putida, and Y. lipolytica. The method precisely yielded absolute concentrations due to the use of internal ${ }^{13} \mathrm{C}$-standards. The addition of the standard during the initial extraction step allowed to compensate for potential concentration changes during sample processing, whereby all thioesters were found rather stable under the given conditions. Notably, the workflow should also deliver robust estimates of free CoA levels. This compound, due its reactive free thiol group, exhibited a certain degree of dimerization into the disulfide (and heterodimers might have been potentially formed with other reduced thiols such as glutathione and mycothiol). All these effects should be compensated by the internal standard (assuming that isotope effects in dimerization were negligible), underling its importance. One should, however, be aware of such side reactions and eventually explore them in more detail, when needed.

Following specific improvement in sampling, sample processing and analytics, we could separate, detect, and quantify CoA thioesters in cell extracts of all studied microbes down to the attomole level within only $10 \mathrm{~min}$ (Fig. 1, 3, and 4). Each microbe revealed a unique CoA thioester spectrum, regarding thioester number, type, and level (Fig. 5a). The method allowed to assess also low abundance CoA thioesters, such as crotonyl-CoA in recombinant $S$. albus (Fig. 4c, d). The wide linear range of $10^{5}-10^{8}$ achieved for quantification (Additional file 1: Fig. S2) appeared crucial to cover the full spectrum of CoA thioesters, which differed more than 10,000-fold in intracellular concentration (Fig. 3d). Statistical analysis of the data, using principal component analysis, revealed that biological triplicates clustered closely for each experiment, independent of the studied strain (Fig. 5b). The concentrations, determined for single CoA esters, were in the same range as values previously observed in these and similar microbes [17-20]. The achieved high reproducibility appears valuable to identify even small phenotypic differences, particularly when considering the general difficulties to obtain precise metabolite and metabolome data [24]. The microbes selected in this study differed significantly in properties that potentially affect the suitability of experimental approaches in metabolomics: cell size and morphology, composition of the cell wall, and presence of specific cellular barriers, such as outer layers or 

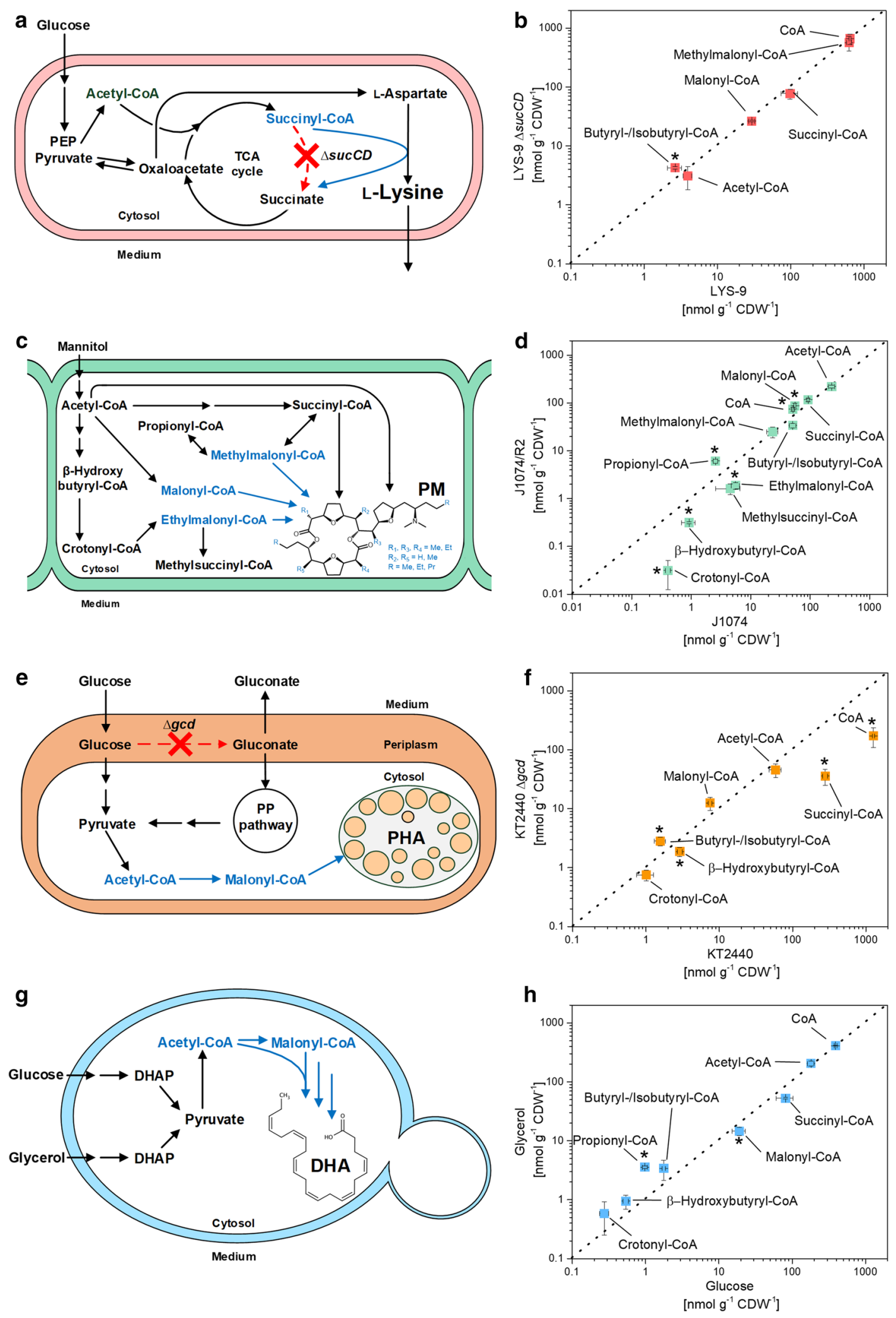

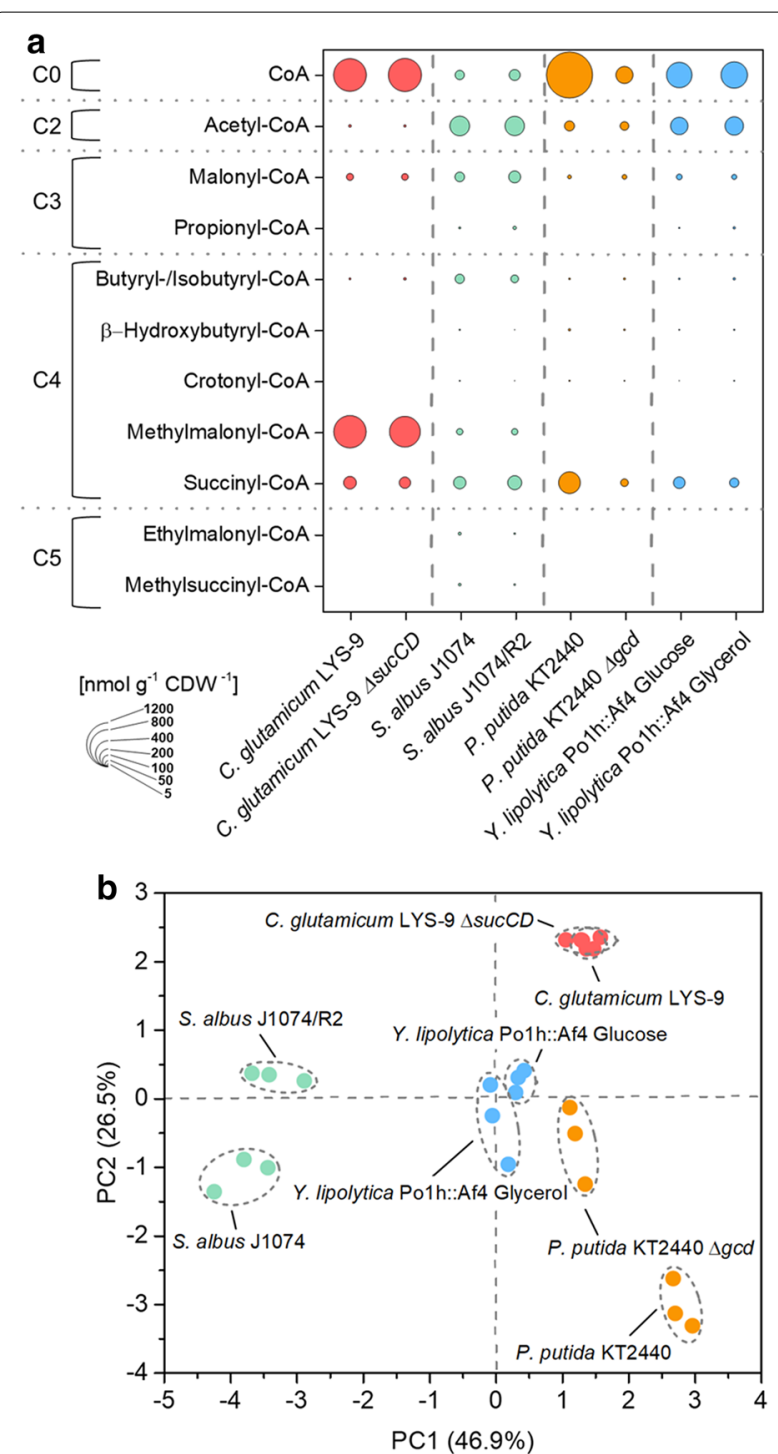

Fig. 5 Spectra of short-chain CoA thioesters and free coenzyme A in different strains of Corynebacterium glutamicum, Streptomyces albus, Pseudomonas putida, and Yarrowia lipolytica. Absolute fingerprints of free CoA and carbon two to carbon five CoA thioesters for eight studied scenarios (a). Principal component analysis of the intracellular spectrum of CoA thioesters and free CoA of all studied scenarios (b). Detailed information on the studied strains is given in the legend to Fig. 3. $n=3$

compartmental membranes [25-27]. The fact that they all could be appropriately analyzed with the same workflow suggests a broad applicability of the method. The possibility to use of one common method for different microbes seems also interesting for automatized screening efforts, which more and more get into focus and benefit from standardized workflows [28].

In the following, the approach was applied to different industrial microbes to demonstrate its potential. As example, we could show that growth of Y. lipolytica on glycerol results in significantly enhanced levels of propionyl-CoA (Fig. 4h). This finding is interesting for the synthesis of odd-chain fatty acids in the yeast, which relies on propionyl-CoA availability and usually requires toxic propionate supplementation or massive strain engineering [29]. In contrast, glucose resulted in a higher amount of malonyl-CoA. In line, this substrate has proven more efficient than glycerol to derive PUFAs, built from this two CoA thioester, in Y. lipolytica [12].

P. putida KT2440 revealed a huge amount of free CoA, more than the other microbes (Fig. 5a), which might be involved in metabolic control, but requires more investigation. A disruption of the periplasmic oxidation route, the major pathway for glucose-breakdown [30] has been previously used to drive PHA synthesis in engineered P. putida [13]. As shown, the mutation did not significantly alter the availability of the PHA building blocks but affected the pools of free CoA and the TCA cycle intermediate succinyl-CoA, suggesting a broader impact on metabolism (Figs. 4e, $\mathrm{f}$, and $5 \mathrm{a}$ ). In addition, the CoA thioester analysis revealed interesting insights into the metabolism of C. glutamicum and S. albus, which are discussed below in more detail.

\section{The succinylase branch of L-lysine biosynthesis efficiently bridges the disrupted TCA cycle in succinate dehydrogenase deficient $C$. glutamicum}

The amino acid L-lysine is an important industrial feed additive, largely produced with C. glutamicum [5]. The TCA cycle competes for carbon with L-lysine biosynthesis but is essential for the aerobic microbe and therefore cannot be eliminated [31]. Increased production, however, can be achieved by flux coupling of the TCA cycle to L-lysine biosynthesis [15]. Succinate dehydrogenase deficient strains cannot convert succinyl-CoA into succinate through the TCA cycle but use the succinylase branch of the L-lysine pathway instead, which results in significantly increased yield (Figs. 2a, b and 4b). One unanswered question so far related to the fact, how the genetic modification affected the availability of succinylCoA for L-lysine biosynthesis [15]. Here, we could show that a block of the TCA cycle at the level of succinate dehydrogenase did not affect the succinyl-CoA pool (Figs. 2 and 4b), and also not that of acetyl-CoA at the entry into the TCA cycle. The TCA cycle mutant and its parent strain exhibited an identical CoA thioester spectrum (Fig. 4b). This demonstrates that the three enzymes of the succinylase branch, succinyl-transferase (DapD), aminotransferase (DapC), and desuccinylase (DapE) [32] fully compensated for the disrupted TCA cycle. An insufficient capacity of this pathway would have otherwise presumably caused an accumulation of succinyl-CoA in 
the mutant. This finding displays a valuable insight into TCA cycle disrupted L-lysine hyper-producing strains. Future profiling of CoA thioesters seems also interesting for other C. glutamicum mutants, in which the L-lysine pathway [33], the TCA cycle [34] and pathways around the CoA thioester metabolism [35-37] have been engineered.

\section{The high abundance of methylmalonyl-CoA in $C$. glutamicum is promising towards heterologous production of complex polyketides}

As shown, methylmalonyl-CoA was the dominating CoA thioester in C. glutamicum (Fig. 4b). From our data, we conclude that methylmalonyl-CoA is formed from succinyl-CoA by methylmalonyl-CoA mutase, eventually as response to TCA cycle activity. Propionyl-CoA, the potentially alternative source for methylmalonyl-CoA via propionyl-CoA carboxylase was proven absent so that this route can be excluded, matching with the fact that propionyl-CoA typically occurs as catabolic intermediate during the degradation of odd-chain fatty acids [38, 39] and branched-chain amino acids [40], not present here.

Methylmalonyl-CoA is a common extender substrate for the biosynthesis of complex polyketides by modular polyketide synthases [41]. The lack of this metabolite has been identified as a barrier to heterologous production of complex polyketides and extensive efforts have been made to install pathways to supply methylmalonylCoA as a building block $[42,43]$. The discovered high abundance of methylmalonyl-CoA is therefore promising for future production of complex polyketides in C. glutamicum, which has been recently demonstrated via functional polyketide synthase expression and 6-methylsalicate biosynthesis in the microbe [36]. For future efforts, the 50-fold excess of methylmalonyl CoA over malonyl-CoA might display an interesting feature, because the relative availability of the two metabolites often impacts the final product structure due to promiscuous enzymes in polyketide synthase assembly lines [41]. Without doubt, the protocol for CoA thioester profiling developed in this work, appears useful for a broad characterization of precursor availability in polyketide producing C. glutamicum mutants.

\section{CoA thioester intermediates from the ethylmalonyl pathway are depleted in pamamycin-producing S. albus and indicate an impact of precursor availability on product formation}

As shown, the CoA thioester spectrum significantly differed between the pamamycin-producing mutant of $S$. albus and the non-producing wildtype (Fig. 3). In particular, intermediates of the ethylmalonyl-CoA pathway [44] were decreased up to more than ten-fold in the producer: $\beta$-hydroxybutyryl-CoA, crotonyl-CoA, ethylmalonyl-CoA, and methylsuccinyl-CoA (Fig. 4d). The formation of pamamycin in the heterologous host obviously consumed more CoA thioester building blocks than were supplied from central metabolism. This could display a bottleneck towards higher titers and deserves further investigation in the future. It was interesting to note that introduction of the heterologous pamamycin pathway perturbed the ratio between malonyl-CoA, methylmalonyl-CoA, and ethylmalonyl-CoA. It was approximately 100:40:10 in the wild type and changed to 100:30:1 in the producer (Fig. 3c, d). The three building blocks compete for incorporation into pamamycin. Unusual polyketide synthases in the assembly line equally accept them as substrates, which leads to 16 pamamycin homologues that differ in their side chains at six positions $[11,45]$. As shown from our data, the dramatically reduced availability of ethylmalonyl-CoA, together with the accumulation of malonyl-CoA, promoted the synthesis of smaller pamamycins. Indeed, $95.7 \%$ of all pamamycin derivatives observed (Pam 579, Pam 593, Pam 607, Pam 621 ) were light ones (had a lower mass), which could be formed without any contribution of ethylmalonyl-CoA. The pamamycins of higher mass (Pam 635, 649 and 663), which required one, two or even more ethylmalonyl-CoA units, became exceedingly rare, based on this effect. It would be interesting to further explore this link in other natural producers, which obviously differ in the spectrum of pamamycin homologues [46-48]. Metabolic engineering of CoA thioester supply appears promising to streamline pamamycin production towards selective derivatives, as proven valuable for other polyketides [18]. Likewise, a variation of bioprocess parameters appears promising to tailor the CoA ester spectrum [49].

\section{Materials and methods Microorganisms}

Strains used in this study were obtained from previous work. This included Streptomyces albus J1074 and its pamamycin producing derivative J1074/R2 [11], the two L-lysine producing strains Corynebacterium glutamicum LYS-9 and LYS-9 $\triangle$ sucCD [15], Pseudomonas putida KT2440 and its mutant KT2440 $\Delta g c d$ [13], and the docosahexaenoic acid (DHA) producing recombinant yeast Yarrowia lipolytica Po1h::Af4 [12]. All strains were maintained as glycerol stocks at $-80{ }^{\circ} \mathrm{C}$.

\section{Media}

Streptomyces albus was kept on mannitol-soy flour (MS) agar containing per liter: $20 \mathrm{~g}$ mannitol, $20 \mathrm{~g}$ soy flour (Schoenenberger Hensel, Magstadt, Germany), and $20 \mathrm{~g}$ agar (Becton-Dickinson, Heidelberg, Germany) [49]. Liquid pre-cultures of $S$. albus were grown in LB broth 
(20 $\mathrm{g} \mathrm{L}^{-1}$, Sigma-Aldrich, Darmstadt, Germany) and main cultures were grown in minimal medium, which contained per liter: $10 \mathrm{~g}$ mannitol, $200 \mathrm{mM}$ potassium

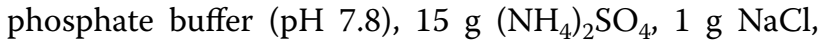
$550 \mathrm{mg} \mathrm{MgCl} 2 * 7 \mathrm{H}_{2} \mathrm{O}, 200 \mathrm{mg} \mathrm{CaCl} 2,30 \mathrm{mg}$ 3,4-dihydroxybenzoic acid, $20 \mathrm{mg} \mathrm{FeSO}$, 2 mg FeCl $3{ }_{3}^{*} 6 \mathrm{H}_{2} \mathrm{O}, 2 \mathrm{mg}$ $\mathrm{MnSO}_{4}{ }^{*} \mathrm{H}_{2} \mathrm{O}, 0.5 \mathrm{mg} \mathrm{ZnSO}{ }_{4}{ }^{*} \mathrm{H}_{2} \mathrm{O}, 0.2 \mathrm{mg} \mathrm{CuCl}{ }_{2}{ }^{*} 2 \mathrm{H}_{2} \mathrm{O}$, $0.2 \mathrm{mg} \mathrm{Na} \mathrm{B}_{4} \mathrm{O}_{7}{ }^{*} 10 \mathrm{H}_{2} \mathrm{O}, 0.1 \mathrm{mg}\left(\mathrm{NH}_{4}\right)_{6} \mathrm{Mo}_{7} \mathrm{O}_{24}{ }^{*} 4 \mathrm{H}_{2} \mathrm{O}$, $1 \mathrm{mg}$ nicotinamide, $1 \mathrm{mg}$ riboflavin, $0.5 \mathrm{mg}$ thiamine hydrochloride, $0.5 \mathrm{mg}$ pyridoxine hydrochloride, $0.2 \mathrm{mg}$ biotin, and $0.1 \mathrm{mg}$ p-aminobenzoic acid. In addition, liquid media were amended with $30 \mathrm{~g} \mathrm{~L}^{-1}$ glass beads (soda-lime glass, $5 \mathrm{~mm}$, Sigma-Aldrich) to avoid cell agglomeration.

Corynebacterium glutamicum was kept on BHI agar (37 $\mathrm{g} \mathrm{L}^{-1}$ BHI, $20 \mathrm{~g} \mathrm{~L}^{-1}$ agar, Becton-Dickinson). Precultures and main cultures of $C$. glutamicum were grown on complex BHI medium and minimal glucose medium, respectively, as described previously [3].

Pseudomonas putida was kept on BHI agar $\left(37 \mathrm{~g} \mathrm{~L}^{-1}\right.$ BHI, $20 \mathrm{~g} \mathrm{~L}^{-1}$ agar, Becton-Dickinson). The mineral M9 medium, used for all liquid cultures, contained per liter: 20 g glucose, $12.8 \mathrm{~g} \mathrm{Na}_{2} \mathrm{HPO}_{4} * 7 \mathrm{H}_{2} \mathrm{O}, 3 \mathrm{~g} \mathrm{KH}_{2} \mathrm{O}_{4}$, $1 \mathrm{~g} \mathrm{NH}_{4} \mathrm{Cl}, 0.5 \mathrm{~g} \mathrm{NaCl}, 0.25 \mathrm{~g} \mathrm{MgSO}_{4} * 7 \mathrm{H}_{2} \mathrm{O}, 6 \mathrm{mg}$ $\mathrm{FeSO}_{4}{ }^{*} 7 \mathrm{H}_{2} \mathrm{O}, 2.7 \mathrm{mg} \mathrm{CaCO}{ }_{3}, 2.0 \mathrm{mg} \mathrm{ZnSO}_{4}{ }^{*} \mathrm{H}_{2} \mathrm{O}, 1.2 \mathrm{mg}$ $\mathrm{MnSO}_{4}{ }^{*} \mathrm{H}_{2} \mathrm{O}, 0.4 \mathrm{mg} \mathrm{CoSO}_{4}{ }^{*} 7 \mathrm{H}_{2} \mathrm{O}, 0.3 \mathrm{mg} \mathrm{CuSO}_{4} * 5 \mathrm{H}_{2} \mathrm{O}$, and $0.1 \mathrm{mg} \mathrm{H}_{3} \mathrm{BO}_{3}$ [13].

Yarrowia lipolytica was incubated on YNB-N5000 agar, which contained per liter: $10 \mathrm{~g}$ glucose, $5 \mathrm{~g}\left(\mathrm{NH}_{4}\right)_{2} \mathrm{SO}_{4}$, $1.7 \mathrm{~g}$ YNB (yeast nitrogen base w/o amino acids and ammonium sulfate, Sigma-Aldrich), and $20 \mathrm{~g}$ agar. All liquid cultures of the yeast were conducted in minimal medium, containing per liter: $10 \mathrm{~g}$ glycerol or $10 \mathrm{~g}$ glucose, $200 \mathrm{mM}$ 2-( $N$-morpholino)ethanesulfonic acid (MES, $\mathrm{pH} 6.8$ ), $5 \mathrm{~g}\left(\mathrm{NH}_{4}\right) 2 \mathrm{SO}_{4}$, and $1.7 \mathrm{~g} \mathrm{YNB}$.

\section{Cultivation in shake flasks}

Liquid cultures were incubated in baffled shake flasks (500 mL, 10\% filling volume) on an orbital shaker (Multitron, Infors AG, Bottmingen, Switzerland, $5 \mathrm{~cm}$ shaking diameter, $230 \mathrm{rpm}, 75 \%$ relative humidity), whereby the temperature was adjusted individually $\left(30{ }^{\circ} \mathrm{C}\right.$ for $P$. putida and C. glutamicum; $28^{\circ} \mathrm{C}$ for S. albus and Y. lipolytica). For each strain, a specific protocol for inoculation and pre-culturing was used to obtain reproducibly growing main cultures. S. albus was incubated on MS agar at $28^{\circ} \mathrm{C}$ for 3 days until sporulation occurred. Spores of a single colony were collected to inoculate the preculture, which was incubated overnight in LB medium. Afterwards, cells were collected $\left(5000 \times g, 25^{\circ} \mathrm{C}, 6 \mathrm{~min}\right)$, resuspended in main culture medium, and used to inoculate the main culture. C. glutamicum was grown overnight on $\mathrm{BHI}$ agar at $30{ }^{\circ} \mathrm{C}$. A single colony was used to inoculate an overnight pre-culture, which was then collected $\left(5,000 \mathrm{xg}, 25^{\circ} \mathrm{C}, 6 \mathrm{~min}\right)$, resuspended in main culture medium, and used to inoculate the main culture. $P$. putida was grown overnight on $\mathrm{M} 9$ agar $\left(30^{\circ} \mathrm{C}\right)$. A single colony served as inoculum for the pre-culture which was then grown overnight, harvested $\left(5,000 \mathrm{xg}, 25^{\circ} \mathrm{C}, 6 \mathrm{~min}\right)$, resuspended in main culture medium, and used to inoculate the main culture. $Y$. lipolytica was grown overnight on YNB-N5000 agar at $28^{\circ} \mathrm{C}$. A single colony was used to inoculate the pre-culture, which was incubated overnight, harvested $\left(5000 \times g, 25{ }^{\circ} \mathrm{C}, 6 \mathrm{~min}\right)$, resuspended in main culture medium and then served as inoculum for the main culture. All growth experiments were conducted as biological triplicate.

\section{Determination of cell concentration}

All investigated microbes were analyzed for their cell dry weight. Cells of $S$. albus were vacuum-filtered using a nitrocellulose filter $(0.2 \mu \mathrm{M}$, Sartorius, Göttingen, Germany), washed twice with $15 \mathrm{~mL}$ deionized water, and gravimetrically analyzed using a moisture analyzer (HB43-S, Mettler-Toledo, Columbus, USA). The parallel measurement of the cell concentration as optical density at $600 \mathrm{~nm}\left(\mathrm{OD}_{600}\right)$ resulted in a correlation factor of $\mathrm{CDW}\left(\mathrm{g} \mathrm{L}^{-1}\right)=0.62 \times \mathrm{OD}_{600}$. The cell dry weight of $C$. glutamicum was inferred from the optical density measurement at $660 \mathrm{~nm}$ as previously described [3]. The cell dry weight of $P$. putida and Y. lipolytica was measured as follows. Cells were collected $\left(15,000 \times g, 4{ }^{\circ} \mathrm{C}, 10 \mathrm{~min}\right)$, washed twice with $15 \mathrm{~mL}$ deionized water, and freezedried. Afterwards, the dry biomass was gravimetrically determined.

\section{Quantification of substrates}

Mannitol and glucose were quantified by HPLC (1260 Infinity Series, Agilent, Darmstadt, Germany) using a Metacarb $87 \mathrm{C}$ column $(300 \times 7.8 \mathrm{~mm}$, Agilent $)$, a Metacarb $87 \mathrm{C}$ guard column $(50 \times 7.8 \mathrm{~mm}$, Agilent), a desalting column (Microguard Deashing Cartridge, Bio-Rad, Munich, Germany), and demineralized water as mobile phase $\left(85^{\circ} \mathrm{C}, 0.6 \mathrm{~mL} \mathrm{~min}{ }^{-1}\right)$. Refraction index measurement was used for detection, and external standards were used for quantification $[2,3]$.

\section{Extraction and quantification of pamamycins}

Prior to analysis, pamamycins were extracted from $S$. albus culture broth. For this purpose, $200 \mu \mathrm{L}$ broth was mixed with $200 \mu \mathrm{L}$ acetone and incubated for $15 \mathrm{~min}$ $(1,000 \mathrm{rpm}$, room temperature, Thermomixer F1.5, Eppendorf, Wesseling, Germany). Afterwards, $200 \mu \mathrm{L}$ ethyl acetate was added, and the mixture was incubated for further $15 \mathrm{~min}$. The organic phase was collected by centrifugation $(20,000 \times g, 5 \mathrm{~min}$, room temperature). 
Subsequently, the solvent mixture was evaporated under nitrogen. The obtained extract was dissolved in methanol and clarified from debris $\left(20,000 \mathrm{xg}, 5 \mathrm{~min}, 4{ }^{\circ} \mathrm{C}\right)$. Afterwards, the different pamamycin derivatives were analyzed using LC-ESI-MS/MS (QTRAP 6500 ${ }^{+}$AB Sciex, Darmstadt, Germany) coupled to an HPLC system (Agilent Infinity 1290 System). In short, the analytes were separated on a C18 column (Vision HT C18 HighLoad, $100 \mathrm{~mm} \times 2 \mathrm{~mm}, 1.5 \mu \mathrm{m}$, Dr. Maisch, AmmerbuchEntringen, Germany) at $45{ }^{\circ} \mathrm{C}$ and a flow rate of $300 \mu \mathrm{L}$ $\min ^{-1}$ (8 $\mathrm{mM}$ ammonium formate in $92 \%$ acetonitrile). Detection was carried out in positive selected ion monitoring (SIM) mode, using the $[\mathrm{M}+\mathrm{H}]^{+}$ion for each pamamycin derivative.

\section{Quantification of L-lysine}

The amino acid L-lysine was quantified using HPLC with pre-column derivatization and fluorescence detection as described before [50]. For quantification, $\alpha$-aminobutyric acid was used as internal standard [2].

\section{Extraction of intracellular CoA thioesters}

A broth sample (approximately $8 \mathrm{mg} C D W$ ) was collected and immediately transferred into a pre-cooled extraction and quenching buffer (95\% acetonitrile, $25 \mathrm{mM}$ formic acid, $-20^{\circ} \mathrm{C}$ ) [21]. The volume ratio was $1: 4$. The obtained solution was thoroughly mixed while cooled on ice for $10 \mathrm{~min}$, and then clarified from debris $\left(15,000 \times g, 4{ }^{\circ} \mathrm{C}, 10\right.$ min). The obtained supernatant was mixed with $10 \mathrm{~mL}$ super cooled deionized water $\left(-2{ }^{\circ} \mathrm{C}\right)$. The cell pellet was twice washed with $8 \mathrm{~mL}$ super cooled deionized water. Afterwards, all supernatants were combined, frozen with liquid nitrogen, freeze-dried, and then re-dissolved in $500 \mu \mathrm{L}$ pre-cooled resuspension buffer $(25 \mathrm{mM}$ ammonium formate, $\mathrm{pH} 3.0,2 \% \mathrm{MeOH}, 4{ }^{\circ} \mathrm{C}$ ) [51]. The buffered extract was filtered (Ultrafree-MC $0.22 \mu \mathrm{m}$, Merck, Millipore, Germany) prior to analysis.

\section{Quantification of CoA thioesters using LC-ESI-MS/MS}

The analysis of CoA thioesters was performed on a triple quadrupole MS (QTRAP $6500^{+}$, AB Sciex, Darmstadt, Germany) coupled to an HPLC system (Agilent Infinity 1290 System). Generally, the injection volume was $10 \mu \mathrm{L}$. Separation of the analytes of interest was conducted on a porous reversed phase column (Gemini C18, $100 \mathrm{~mm}$ $\times 4.6 \mathrm{~mm}, 3 \mu \mathrm{m}, 110 \AA$, Phenomenex, Aschaffenburg, Germany) at $40{ }^{\circ} \mathrm{C}$ using a gradient of formic acid (50 mM, adjusted to $\mathrm{pH} 8.1$ with ammonium hydroxide $25 \%$ in $\mathrm{H}_{2} \mathrm{O}$, eluent $\mathrm{A}$ ) and methanol (eluent $\mathrm{B}$ ) at a flow rate of $600 \mu \mathrm{L} \mathrm{min}{ }^{-1}$. The fraction of eluent $B$ was as follows: $0-12 \mathrm{~min}, 0-15 \% \mathrm{~B} ; 12-16 \mathrm{~min}, 15-100 \% \mathrm{~B}$; 16-18 min, $100 \% \mathrm{~B} ; 18-20 \mathrm{~min}, 100-0 \% \mathrm{~B} ; 20-25 \mathrm{~min}$, $0 \% \mathrm{~B}$. Initial tests were further done, using a smaller column geometry and pore size (Gemini C18, $100 \mathrm{~mm}$ $\times 2.1 \mathrm{~mm}, 1.5 \mu \mathrm{m}, 110 \AA$, Phenomenex), using the same gradient, but a reduced flow rate of $300 \mu \mathrm{L} \mathrm{min}{ }^{-1}$. In addition (and finally used in the optimized workflow), a core-shell reversed phase column (Kinetex XB-C18, $100 \times 2.1 \mathrm{~mm}, 2.6 \mu \mathrm{m}, 100 \AA$, Phenomenex) was applied at $40{ }^{\circ} \mathrm{C}$, using a gradient of formic acid $(50 \mathrm{mM}$, adjusted to $\mathrm{pH} 8.1$ with ammonium hydroxide $25 \%$ in $\mathrm{H}_{2} \mathrm{O}$, eluent A) and methanol (eluent B) at a flow rate of $300 \mu \mathrm{L}$ $\mathrm{min}^{-1}$. The fraction of eluent $B$ was as follows: $0-7 \mathrm{~min}$, 0-10\% B; 7-10 min, 10-100\% B; 10-11 min, $100 \% \mathrm{~B}$; 11-12 min, $100-0 \%$ B; $12-15$ min, 0\% B. During the first 3 min of the analysis, the outflow from the chromatographic column was discharged to minimize the entry of salts from samples into the mass spectrometer. The individual CoA thioesters were detected using multiple reaction monitoring (MRM), involving the corresponding parent ion and its respective daughter ion (Additional file 1: Table S1). Further instrument settings were as follows: curtain gas, $35 \mathrm{psi}$; collision gas flowrate, medium; ion spray voltage, $4.5 \mathrm{kV}$; temperature, $400{ }^{\circ} \mathrm{C}$; ion source gas, $60 \mathrm{psi}$; and entrance potential, $10 \mathrm{~V}$. The declustering potential, the collision energy and the collision cell exit potential were optimized individually for each CoA thioester using synthetic standards. Acetyl-CoA, propionylCoA, succinyl-CoA, methylmalonyl-CoA, and free CoA were purchased (Sigma-Aldrich), whereas malonyl-CoA, $\beta$-hydroxybutyryl-CoA, butyryl-CoA, isobutyryl-CoA, crotonyl-CoA, methylsuccinyl-CoA and ethylmalonylCoA were chemo-enzymatically synthesized as previously described [7].

\section{Absolute quantification of $\mathrm{CoA}$ thioesters using ${ }^{13} \mathrm{C}$-labeled extracts}

Absolute quantification of CoA thioesters was conducted using the MIRACLE approach [52]. For this purpose, ${ }^{13} \mathrm{C}$-labeled cell extracts were used as internal standard, whereby an individual standard was produced for each microbe. For this purpose, the different organisms were grown on ${ }^{13} \mathrm{C}$-enriched substrates, i.e. the naturally labeled carbon source was replaced by an equimolar amount of the $\left[\mathrm{U}_{-}{ }^{13} \mathrm{C}\right]$ enriched isomer: 99\% $\left[{ }^{13} \mathrm{C}_{6}\right]$ D-mannitol (S. albus), 99\% $\left[{ }^{13} \mathrm{C}_{6}\right] \mathrm{D}$-glucose (P. putida and C. glutamicum), and 99\% $\left[{ }^{13} \mathrm{C}_{3}\right]$ D-glycerol ( $Y$. lipolytica). The ${ }^{13} \mathrm{C}$ tracer substrates were obtained from Cambridge Isotope Laboratories (Tewksbury, MA, USA). For each microbe, the second pre-culture and the main culture was conducted in the ${ }^{13} \mathrm{C}$-enriched minimal medium. In each case, the inoculum size was below $1 \%$ of the later harvested cell concentration to finally provide fully ${ }^{13} \mathrm{C}$ enriched cell extracts as standard and exclude potential interference [27]. The culture broth from the main ${ }^{13} \mathrm{C}$ culture was 
extracted during the late exponential growth phase, using the extraction protocol described above. After freeze-drying and re-suspension, the ${ }^{13} \mathrm{C}$ extract was stored as aliquots at $-80^{\circ} \mathrm{C}$. The level of the individual CoA thioesters in each ${ }^{13} \mathrm{C}$ extract was precisely quantified using the synthetic standards and corresponding instrument settings (Additional file 1: Tables S1 and S2). During later analysis, an appropriate volume of ${ }^{13} \mathrm{C}$ extract (of the respective microbe) was thawn on ice, and then simultaneously added with the sample into the quenching solution. This protocol allowed to to infer absolute metabolite levels and to take any eventual changes during sample processing into account.

\section{Principal component analysis}

Principle component analysis (PCA) was performed using the ClustVis web tool [53].

\section{Supplementary information}

Supplementary information accompanies this paper at https://doi. org/10.1186/s12934-020-01413-1.

Additional file 1: Figure S1. LC-MS chromatogram of a synthetic CoA thioester standard using a porous organo-silica reversed phase column $(100 \times 2.1 \mathrm{~mm}, 1.5 \mu \mathrm{m})$ for the chromatographic separation. Co-eluting analytes were distinguished by a different specific mass-to-charge ratio $(\mathrm{m} / \mathrm{z})$. Figure S2. Calibration curves for different CoA thioesters using LC-MS/MS analysis. Table S1. Instrumental settings for LC-MS/MS analysis of CoA thioesters. The declustering potential (DP), the collision energy (CE) and the cell exit potential (CXP) were individually tuned for each CoA thioester. The parent ion reflects the positive proton adduct $[\mathrm{M}+\mathrm{H}]^{+}$, except for the CoA homodimer (CoA-S-S-CoA), where the parent ion was $[\mathrm{M}+2 \mathrm{H}]^{2+}$. In each case, the daughter ion reflects the positive proton adduct after neutral loss of $507(\mathrm{~m} / \mathrm{z})$. Table $\mathbf{S 2}$. Instrumental settings for LC-MS/MS analysis of fully ${ }^{13} \mathrm{C}$-labeled CoA thioesters used as internal standard for absolute CoA thioesters quantification. The respective mass of the fully labeled parent ion was determined by adding the number of carbon atoms to the monoisotopic mass of the non-labelled parent ion (Table S1). The mass of each daughter ion was then calculated by subtraction of $m / z 517$ form this value, considering the neutral loss of a fragment with ten ${ }^{13} \mathrm{C}$ atoms.

\section{Authors' contributions}

BV synthesized CoA thioesters. LG, MK and MF developed the CoA thioester extraction and analytical protocol. LG produced the ${ }^{13} \mathrm{C}$ labeled extracts and conducted cultivation of S. albus, C. glutamicum, and P. putida. SJ performed cultivation of $Y$. lipolytica. LG conducted thioester analysis. CW conceived and structured the work. LG, JB and CW wrote the first draft of the manuscript. All authors critically commented and improved the manuscript. All authors read and approved the final manuscript.

\section{Funding}

Open access funding providedby Projekt DEAL. This work was supported by the German Ministry of Education and Research (BMBF) through the Grants MyBio (FKZ 031B034A) and MYXO4PUFA (FKZ 031B0346B) and by the German Research Foundation (FKZ INST 256/418-1). Further support was provided by the Max Planck Society and the LOEWE program (Landes-Offensive zur Entwicklung wissenschaftlich-ökonomischer Exzellenz) of the state of Hessen within the framework of the MegaSyn Research Cluster. The funding bodies did not contribute to study design, data collection, analysis, and interpretation, or writing of the manuscript.

\section{Availability of data and materials}

The dataset(s) supporting the conclusions of this article are all included within the article.

\section{Ethics approval and consent to participate}

Not applicable. The manuscript does not contain data collected from humans or animals.

\section{Consent for publication \\ Not applicable.}

\section{Competing interests}

The authors declare that they have no competing interests.

\section{Author details}

${ }^{1}$ Institute of Systems Biotechnology, Saarland University, Saarbrücken, Germany. ${ }^{2}$ Max Planck Institute for Terrestrial Microbiology, Marburg, Germany.

Received: 4 May 2020 Accepted: 20 July 2020

Published online: 10 August 2020

\section{References}

1. Becker J, Wittmann C. From systems biology to metabolically engineered cells-an omics perspective on the development of industrial microbes. Curr Opin Microbiol. 2018:45:180-8

2. Hoffmann SL, Jungmann L, Schiefelbein S, Peyriga L, Cahoreau E, Portais JC, Becker J, Wittmann C. Lysine production from the sugar alcohol mannitol: design of the cell factory Corynebacterium glutamicum SEA-3 through integrated analysis and engineering of metabolic pathway fluxes. Metab Eng. 2018;47:475-87.

3. Rohles CM, Giesselmann G, Kohlstedt M, Wittmann C, Becker J. Systems metabolic engineering of Corynebacterium glutamicum for the production of the carbon-5 platform chemicals 5-aminovalerate and glutarate. Microb Cell Fact. 2016;15:154.

4. Rohles CM, Gläser L, Kohlstedt M, Giesselmann G, Pearson S, del Campo A, Becker J, Wittmann C. A bio-based route to the carbon-5 chemical glutaric acid and to bionylon-6,5 using metabolically engineered Corynebacterium glutamicum. R Soc Chem Green Chem. 2018;20:4662-74.

5. Becker J, Rohles CM, Wittmann C. Metabolically engineered Corynebacterium glutamicum for bio-based production of chemicals, fuels, materials, and healthcare products. Metab Eng. 2018;50:122-41.

6. Veras HCT, Campos CG, Nascimento IF, Abdelnur PV, Almeida JRM, Parachin NS. Metabolic flux analysis for metabolome data validation of naturally xylose-fermenting yeasts. BMC Biotechnol. 2019:19:58.

7. Peter DM, Vogeli B, Cortina NS, Erb TJ. A chemo-enzymatic road map to the synthesis of CoA esters. Molecules. 2016;21:517.

8. Zimmermann M, Thormann V, Sauer U, Zamboni N. Nontargeted profiling of coenzyme A thioesters in biological samples by tandem mass spectrometry. Anal Chem. 2013;85:8284-90.

9. Kanehisa M, Goto S. KEGG: kyoto encyclopedia of genes and genomes. Nucleic Acids Res. 2000;28:27-30.

10. Kanehisa M, Sato Y, Furumichi M, Morishima K, Tanabe M. New approach for understanding genome variations in KEGG. Nucleic Acids Res. 2019;47:D590-5.

11. Rebets Y, Brotz E, Manderscheid N, Tokovenko B, Myronovskyi M, Metz P, Petzke L, Luzhetskyy A. Insights into the pamamycin biosynthesis. Angew Chem. 2015;54:2280-4.

12. Gemperlein K, Dietrich D, Kohlstedt M, Zipf G, Bernauer HS, Wittmann C, Wenzel SC, Müller R. Polyunsaturated fatty acid production by Yarrowia lipolytica employing designed myxobacterial PUFA synthases. Nat Commun. 2019;10:4055.

13. Poblete-Castro I, Binger D, Rodrigues A, Becker J, Martins Dos Santos VA Wittmann C. In-silico-driven metabolic engineering of Pseudomonas putida for enhanced production of poly-hydroxyalkanoates. Metab Eng. 2013;15:113-23.

14. Kang A, Lee TS. Converting sugars to biofuels: ethanol and beyond Bioengineering. 2015;2:184-203.

15. Kind S, Becker J, Wittmann C. Increased lysine production by flux coupling of the tricarboxylic acid cycle and the lysine biosynthetic 
pathway-metabolic engineering of the availability of succinyl-CoA in Corynebacterium glutamicum. Metab Eng. 2013;15:184-95.

16. Schada von Borzyskowski L, Sonntag F, Poschel L, Vorholt JA, Schrader J, Erb TJ, Buchhaupt M. Replacing the ethylmalonyl-CoA pathway with the glyoxylate shunt provides metabolic flexibility in the central carbon metabolism of Methylobacterium extorquens AM1. ACS Synth Biol. 2018;7:86-97.

17. Huang YY, Jian XX, Lv YB, Nian KQ, Gao Q, Chen J, Wei LJ, Hua Q. Enhanced squalene biosynthesis in Yarrowia lipolytica based on metabolically engineered acetyl-CoA metabolism. J Biotechnol. 2018;281:106-14.

18. Lu C, Zhang X, Jiang M, Bai L. Enhanced salinomycin production by adjusting the supply of polyketide extender units in Streptomyces albus. Metab Eng. 2016;35:129-37.

19. Martinez-Garcia E, Nikel PI, Aparicio T, de Lorenzo V. Pseudomonas 2.0: genetic upgrading of $P$. putida KT2440 as an enhanced host for heterologous gene expression. Microbial cell factories. 2014;13:159.

20. Botella L, Lindley ND, Eggeling L. Formation and metabolism of methylmalonyl coenzyme A in Corynebacterium glutamicum. J Bacteriol. 2009;191:2899-901.

21. Peyraud R, Kiefer P, Christen P, Massou S, Portais JC, Vorholt JA. Demonstration of the ethylmalonyl-CoA pathway by using ${ }^{13} \mathrm{C}$ metabolomics. Proc Natl Acad Sci USA. 2009;106:4846-51.

22. Seifar RM, Ras C, Deshmukh AT, Bekers KM, Suarez-Mendez CA, da Cruz AL, van Gulik WM, Heijnen JJ. Quantitative analysis of intracellular coenzymes in Saccharomyces cerevisiae using ion pair reversed phase ultra high performance liquid chromatography tandem mass spectrometry. J Chromatogr A. 2013:1311:115-20.

23. Park JW, Jung WS, Park SR, Park BC, Yoon YJ. Analysis of intracellular short organic acid-coenzyme A esters from actinomycetes using liquid chromatography-electrospray ionization-mass spectrometry. Journal of mass spectrometry: JMS. 2007;42:1136-47.

24. Rost LM, Brekke Thorfinnsdottir L, Kumar K, Fuchino K, Eide Langorgen I, Bartosova Z, Kristiansen KA, Bruheim P. Absolute quantification of the central carbon metabolome in eight commonly applied prokaryotic and eukaryotic model systems. Metabolites. 2020;10:74.

25. Bolten CJ, Kiefer P, Letisse F, Portais JC, Wittmann C. Sampling for metabolome analysis of microorganisms. Anal Chem. 2007;79:3843-9.

26. Wittmann C, Krömer JO, Kiefer P, Binz T, Heinzle E. Impact of the cold shock phenomenon on quantification of intracellular metabolites in bacteria. Anal Biochem. 2004;327:135-9.

27. Wittmann C. Fluxome analysis using GC-MS. Microb Cell Fact. 2007;6:6.

28. Weber RJM, Lawson TN, Salek RM, Ebbels TMD, Glen RC, Goodacre R, Griffin JL, Haug K, Koulman A, Moreno P, et al. Computational tools and workflows in metabolomics: an international survey highlights the opportunity for harmonisation through Galaxy. Metabolomics. 2017;13:12.

29. Park YK, Ledesma-Amaro R, Nicaud JM. De novo biosynthesis of oddchain fatty acids in Yarrowia lipolytica enabled by modular pathway engineering. Front Bioeng Biotechnol. 2019;7:484.

30. Kohlstedt M, Wittmann C. GC-MS-based (13)C metabolic flux analysis resolves the parallel and cyclic glucose metabolism of Pseudomonas putida KT2440 and Pseudomonas aeruginosa PAO1. Metab Eng. 2019;54:35-53.

31. Becker J, Klopprogge C, Schroder H, Wittmann C. Metabolic engineering of the tricarboxylic acid cycle for improved lysine production by Corynebacterium glutamicum. Appl Environ Microbiol. 2009;75:7866-9.

32. Sonntag K, Eggeling L, De Graaf AA, Sahm H. Flux partitioning in the split pathway of lysine synthesis in Corynebacterium glutamicum. Quantification by 13C- and 1H-NMR spectroscopy. Eur J Biochem. 1993:213:1325-31.

33. Becker J, Zelder O, Haefner S, Schröder H, Wittmann C. From zero to hero-design-based systems metabolic engineering of Corynebacterium glutamicum for L-lysine production. Metab Eng. 2011;13:159-68.

34. Zhang Y, Shang X, Wang B, Hu Q, Liu S, Wen T. Reconstruction of tricarboxylic acid cycle in Corynebacterium glutamicum with a genomescale metabolic network model for trans-4-hydroxyproline production. Biotechnol Bioeng. 2019;116:99-109.

35. Milke L, Kallscheuer N, Kappelmann J, Marienhagen J. Tailoring Corynebacterium glutamicum towards increased malonyl-CoA availability for efficient synthesis of the plant pentaketide noreugenin. Microb Cell Fact. 2019;18:71.
36. Kallscheuer N, Kage H, Milke L, Nett M, Marienhagen J. Microbial synthesis of the type I polyketide 6-methylsalicylate with Corynebacterium glutamicum. Appl Microbiol Biotechnol. 2019;103:9619-31.

37. Chang Z, Dai W, Mao Y, Cui Z, Wang Z, Chen T. Engineering Corynebacterium glutamicum for the efficient production of 3-hydroxypropionic acid from a mixture of glucose and acetate via the malonyl-CoA pathway. Catalysts. 2020;10:203.

38. Zhang YQ, Brock M, Keller NP. Connection of propionyl-CoA metabolism to polyketide biosynthesis in Aspergillus nidulans. Genetics. 2004;168:785-94.

39. Dolan SK, Wijaya A, Geddis SM, Spring DR, Silva-Rocha R, Welch M. Loving the poison: the methylcitrate cycle and bacterial pathogenesis. Microbiology. 2018;164:251-9.

40. Xu Z, Liu Y, Ye BC. PccD regulates branched-chain amino acid degradation and exerts a negative effect on erythromycin production in Saccharopolyspora erythraea. Appl Environ Microbiol. 2018;84:e00049-18.

41. Helfrich EJ, Piel J. Biosynthesis of polyketides by trans-AT polyketide synthases. Nat Prod Rep. 2016:33:231-316.

42. Dayem LC, Carney JR, Santi DV, Pfeifer BA, Khosla C, Kealey JT. Metabolic engineering of a methylmalonyl-CoA mutase-epimerase pathway for complex polyketide biosynthesis in Escherichia coli. Biochemistry. 2002:41:5193-201.

43. Gross F, Ring MW, Perlova O, Fu J, Schneider S, Gerth K, Kuhlmann S, Stewart AF, Zhang Y, Müller R. Metabolic engineering of Pseudomonas putida for methylmalonyl-CoA biosynthesis to enable complex heterologous secondary metabolite formation. Chem Biol. 2006;13:1253-64.

44. Wang J, Wang C, Song K, Wen J. Metabolic network model guided engineering ethylmalonyl-CoA pathway to improve ascomycin production in Streptomyces hygroscopicus var. ascomyceticus. Microb Cell Fact. 2017;16:169.

45. Kuhl M, Gläser L, Rebets Y, Rückert C, Sarkar N, Hartsch T, Kalinowksi J, Luzhetskyy A, Wittmann C: Microparticles globally reprogram the metabolism of Streptomyces albus towards accelerated morphogenesis, streamlined carbon core metabolism and enhanced production of the antituberculosis polyketide pamamycin. Biotechnol Bioeng 2020, submitted.

46. Natsume M, Tazawa J, Yagi K, Abe H, Kondo S, Marumo S. Structure-activity relationship of pamamycins: effects of alkyl substituents. J Antibiotics. 1995;48:1159-64.

47. Kozone I, Chikamoto N, Abe H, Natsume M. De-N-methylpamamycin593A and B, new pamamycin derivatives isolated from Streptomyces alboniger. J Antibiotics. 1999;52:329-31.

48. Natsume M, Yasui K, Kondo S, Marumo S. The structure of four new pamamycin homologues isolated from Streptomyces alboniger. Tetrahedron Lett. 1991;32:3087-90.

49. Kieser T, Bibb M, Buttner M, Chater K, Hopwood D. Practical streptomyces genetics John Innes Foundation. United Kingdom: Norwich; 2000.

50. Krömer JO, Fritz M, Heinzle E, Wittmann C. In vivo quantification of intracellular amino acids and intermediates of the methionine pathway in Corynebacterium glutamicum. Anal Biochem. 2005;340:171-3.

51. Schneider K, Peyraud R, Kiefer P, Christen P, Delmotte N, Massou S, Portais JC, Vorholt JA. The ethylmalonyl-CoA pathway is used in place of the glyoxylate cycle by Methylobacterium extorquens AM1 during growth on acetate. J Biol Chem. 2012;287:757-66.

52. Mashego MR, Wu L, Van Dam JC, Ras C, Vinke JL, Van Winden WA, Van GulikWM, Heijnen JJ. MIRACLE: mass isotopomer ratio analysis of $\mathrm{U}-{ }^{13} \mathrm{C}$-labeled extracts. A new method for accurate quantification of changes in concentrations of intracellular metabolites. Biotechnol Bioeng. 2004:85:620-8.

53. Metsalu T, Vilo J. ClustVis: a web tool for visualizing clustering of multivariate data using Principal Component Analysis and heatmap. Nucleic Acids Res. 2015;43:W566-70.

\section{Publisher's Note}

Springer Nature remains neutral with regard to jurisdictional claims in published maps and institutional affiliations. 